\title{
Article
}

\section{The Prognostic Role of Early Skeletal Muscle Mass Depletion in Multimodality Management of Patients with Advanced Gastric Cancer Treated with First Line Chemotherapy: A Pilot Experience from Modena Cancer Center}

\author{
Margherita Rimini ${ }^{1}$, Annarita Pecchi ${ }^{2}$ (D) Francesco Prampolini ${ }^{2}{ }^{(1)}$, Chiara Bussei $^{3}$, Massimiliano Salati ${ }^{1}$, \\ Daniela Forni ${ }^{2}$, Francesca Martelli ${ }^{2}$, Filippo Valoriani ${ }^{3}{ }^{10}$, Fabio Canino ${ }^{1}$, Alessandro Bocconi ${ }^{1}$, \\ Fabio Gelsomino ${ }^{1}{ }^{(0)}$, Linda Reverberi ${ }^{3}$, Stefania Benatti ${ }^{1}$, Federico Piacentini ${ }^{1}{ }^{1}$, Renata Menozzi ${ }^{3}$, \\ Massimo Dominici ${ }^{1}$, Gabriele Luppi ${ }^{1}$ and Andrea Spallanzani ${ }^{1, *(1)}$
}

1 Division of Oncology, Department of Oncology and Hematology, University Hospital of Modena, 41122 Modena, Italy; margherita.rimini@gmail.com (M.R.); massisalati@gmail.com (M.S.); fabiocanino05@gmail.com (F.C.); alessandro.bocconi@gmail.com (A.B.); fabiogelsomino83@yahoo.it (F.G.); stefania.benatti@unimore.it (S.B.); federico.piacentini@unimore.it (F.P.); massimo.dominici@unimore.it (M.D.); luppi.gabriele@aou.mo.it (G.L.)

check for updates

Citation: Rimini, M.; Pecchi, A.; Prampolini, F.; Bussei, C.; Salati, M.; Forni, D.; Martelli, F.; Valoriani, F.; Canino, F.; Bocconi, A.; et al. The Prognostic Role of Early Skeletal Muscle Mass Depletion in Multimodality Management of Patients with Advanced Gastric Cancer Treated with First Line Chemotherapy: A Pilot Experience from Modena Cancer Center. J. Clin Med. 2021, 10, 1705. https:// doi.org/10.3390/jcm10081705

Academic Editor: Angelica Petrillo

Received: 2 March 2021

Accepted: 12 April 2021

Published: 15 April 2021

Publisher's Note: MDPI stays neutral with regard to jurisdictional claims in published maps and institutional affiliations.

Copyright: (c) 2021 by the authors. Licensee MDPI, Basel, Switzerland. This article is an open access article distributed under the terms and conditions of the Creative Commons Attribution (CC BY) license (https:// creativecommons.org/licenses/by/ $4.0 /)$.
2 Department of Radiology, University Hospital of Modena, 41122 Modena, Italy; pecchi.annarita@aou.mo.it (A.P.); prampolini.francesco@aou.mo.it (F.P.); danielaforni92@gmail.com (D.F.); francesca.martelli1992@gmail.com (F.M.)

3 Division of Metabolic Disease and Clinical Nutrition, University Hospital of Modena, 41122 Modena, Italy; bussei.chiara@aou.mo.it (C.B.); valorianifilippo@gmail.com (F.V.); reverberi.linda@policlinico.mo.it (L.R.); menozzi.renata@aou.mo.it (R.M.)

* Correspondence: andrea.spallanzani@gmail.com; Tel.: +39-05-9422-3310

\begin{abstract}
Background: Few data about the link between nutritional status and survival are available in the metastatic gastric cancer (GC) setting. The aim of this work was to evaluate the prognostic role of tissue modifications during treatment and the benefit of a scheduled nutritional assessment in this setting. Methods: Clinical and laboratory variables of 40 metastatic GC patients treated at Modena Cancer Center were retrieved: 20 received a nutritional assessment on the oncology's discretion, the other 20 received a scheduled nutritional assessment at baseline and every 2-4 weeks. Anthropometric parameters were calculated on Computed Tomography (CT) images at the baseline and after 3 months of chemotherapy. Results: A correlation between baseline Eastern Cooperative Oncology Group Performance Status (ECOG PS), Lymphocyte to Monocyte Ratio (LMR), C-reactive protein (PCR), Prognostic Nutritional Index (PNI) and Overall survival (OS) was highlighted. Among the anthropometric parameters, early skeletal muscle mass depletion (ESMMD) $>10 \%$ in the first months of treatment significantly impacted on mOS $(p=0.0023)$. A link between ESMMD and baseline $\mathrm{LDH}>460 \mathrm{U} / \mathrm{L}$, baseline $\mathrm{CRP}>2.2 \mathrm{mg} / \mathrm{dL}$ and weight decrease during treatment emerged. Patients evaluated with a nutritional scheduled support experienced a mean gain in subcutaneous and visceral fat of $11.4 \%$ and $10.21 \%$, respectively. Conclusion: We confirm the prognostic impact of ESMMD $>10 \%$ during chemotherapy in metastatic GC. The prognostic role of a scheduled nutritional assessment deserves further confirmation in large prospective trials.
\end{abstract}

Keywords: gastric cancer; sarcopenia; nutritional status

\section{Introduction}

The prognosis of patients with advanced gastric cancer is still poor due to the absence of potentially curative options [1]. Palliative chemotherapy improves survival and quality of life (QoL) compared to best supportive care both in first and second line setting [2]. In recent years, the development of new drugs alone or in combination with chemotherapy, helped to raise the bar of median overall survival over 12 months at the expense of increased 
treatment related toxicities [3,4]. The introduction of anti-HER2 treatment in first such as the development of ramucirumab alone or in combination with chemotherapy in second line provided nearly 14-16 months of median overall survival in patients with new diagnosis of metastatic gastric cancer [5-7]. In fact, alongside progress in the pharmacological field, the target has moved to an adequate patient selection. The research of clinic-pathological prognostic and predictive factors is one of the main objectives of prospective studies and retrospective analysis $[8,9]$.

Some prognostic scores have been developed combining inflammation-related and nutrition-related markers, such as the neutrophil-to-lymphocyte ratio (NLR) platelets to Lymphocyte ratio (PLR), lymphocyte to monocyte ratio (LMR) and prognostic nutritional index (PNI): the prognostic role of these parameters and other factors such as performance status and neoplastic markers (CEA and CA 19.9) is well known in patients with advanced gastric cancer [10,11].

Almost two decades ago it was identified the close link between malnutrition and survival and the impact of sarcopenia on tolerance to chemotherapy, longer hospitalization, quality of life and mortality, but to date, many malnourished cancer patients still receive inadequate nutritional support, mainly due to the poor awareness of the problem and inefficient collaboration between oncologists and clinical nutritionists [12].

An adequate evaluation of patients' nutritional status in metastatic setting cannot ignore the assessment of skeletal muscle mass and skeletal muscle density using computed tomography scan. Previous studies in various types of cancer highlighted the strong association between survival, treatment toxicities and the amount of muscle and adipose tissue at diagnosis such as its modification during chemotherapy [13-16].

A recent metanalysis focused on the effects of dietary interventions on nutritional status of gastric cancer patients undergoing gastrectomy, but few data are available in patients with metastatic gastric cancer treated with palliative chemotherapy [17].

The aim of our work is to evaluate the incidence of sarcopenia such as the prognostic and predictive role of muscle and visceral tissue modifications during the first 3 months of chemotherapy in patients with metastatic gastric cancer. In a subsequent pivotal analysis, we matched two different group of patients to evaluate the role of an adequate nutritional support during palliative chemotherapy.

\section{Materials and Methods}

Patients with recurrent or metastatic gastric cancer who received fluoropyrimidines and platinum based first-line chemotherapy in Modena Cancer Center from November 2015 through December 2019 were retrospectively studied. All patients had histologically proven adenocarcinoma of stomach with at least one metastatic lesion as confirmed by diagnostic imaging. Computed Tomography (CT) scan was performed every $2-4$ months in most patients to evaluate treatment efficacy. The study was approved by local Ethic Committee ( $\left.n^{\circ} 427 / 2019 / \mathrm{OSS} / \mathrm{AOUMO}\right)$. All alive patients provided written informed consent.

Clinical and laboratory data were reported from the hospital electronic medical database at diagnosis and first $\mathrm{CT}$ re-evaluation including the following variables: age, gender, performance status (ECOG), height, weight, Body-Mass Index (BMI), blood count, neutrophil/lymphocyte ratio (NLR), platelets/lymphocyte ratio (PLR), lymphocyte/monocyte ratio (LMR), systemic inflammatory index (SII) lactate dehydrogenase (LDH), C-reactive protein (CRP), albumin, Sodium $\left(\mathrm{Na}^{+}\right)$, Potassium $\left(\mathrm{K}^{+}\right)$, CEA, CA 19.9 and prognostic nutritional index (PNI).

In the second part of the work, we searched for differences in terms of clinical, anthropometric and survival outcomes between the first group of patients which received a nutritional evaluation at oncology's discretion and the second group of patients which received a standardized nutritional evaluation at the baseline and then every $2-4$ weeks during treatment. 
The nutritional evaluation was defined as an individualized counselling aimed to collecting data about the dietary intake, usual dietary pattern, intolerances or food aversions, digestive difficulties, patients' psychological status, autonomy and need for help in the act of eating. In addition, a symptom assessment was included in the nutritional evaluation. Each nutritional assessment resulted in dietary advice and, if necessary, in prescription of oral implementations.

\subsection{Body Composition Parameter Measurements}

All patients included in the study underwent CT scan at the time of diagnosis and after 2-4 months, as part of the diagnostic and therapeutic path planned by the cancer center.

CT exams were performed at our hospital using a 64-slice CT scanner (Lightspeed VCT, GE Healthcare, Milwaukee, WI, USA).

Baseline and follow-up CT examinations were loaded on an Advantage Workstation (VolumeShare 7, GE Healthcare, Milwaukee, WI, USA) and non-contrast images at the level of the third lumbar vertebra (L3) were used for reconstructions and measurements of quantitative and qualitative body composition parameters.

According to literature, skeletal muscle cross-sectional areas including the psoas, paraspinal muscles and abdominal muscles were identified and quantified using the preestablished HU thresholds for muscle (HU-30 to 150), whereas subcutaneous and visceral adipose cross-sectional areas were quantified using $\mathrm{HU}$ thresholds for fat tissue (HU-150 to-30) (Figure 1).
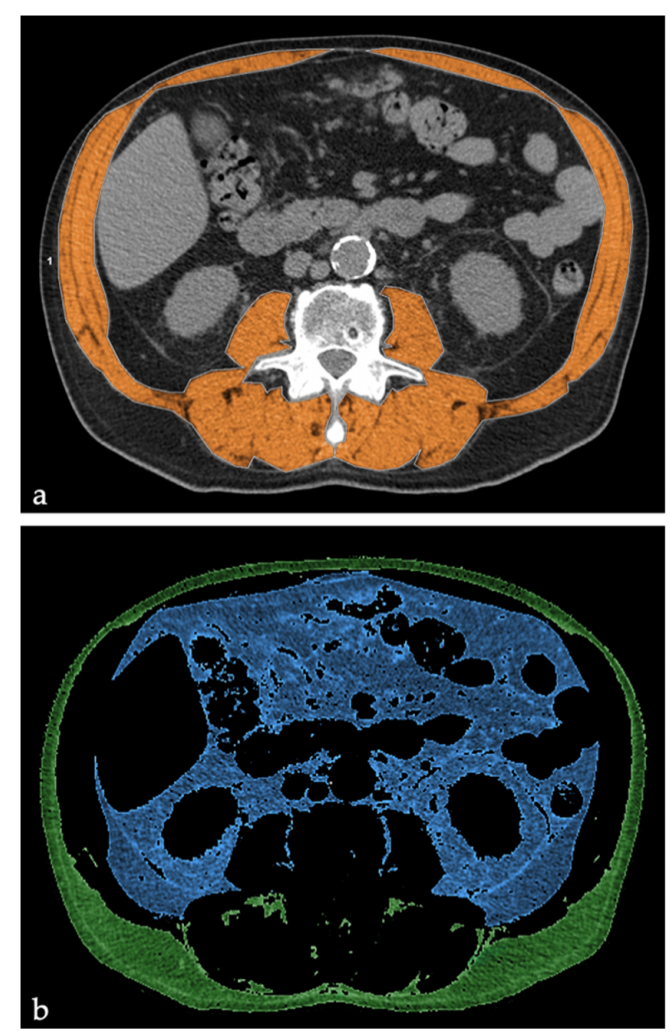

Figure 1. Measurement of body composition parameters with cross-sectional computed tomography (CT) images at the level of third lumbar vertebra. (a) Skeletal muscle area (b) Subcutaneous and Visceral fat areas.

The skeletal muscle mass index $\left(\mathrm{SMI}, \mathrm{cm}^{2} / \mathrm{m}^{2}\right)$ was calculated by dividing these skeletal muscle areas by height squared and similarly visceral fat index (VFI, $\mathrm{cm}^{2} / \mathrm{m}^{2}$ ) and subcutaneous fat index $\left(\mathrm{SFI}, \mathrm{cm}^{2} / \mathrm{m}^{2}\right.$ ) were calculated by normalizing each fat area for height. 
Total adipose index (TAI) was calculated by adding SFI + VFI.

Relative changes of body composition occurred in the period between baseline and follow-up CT scans were also quantified for each patient by calculating delta $(\Delta)$ parameters: $\Delta$ SMI (defined also as ESMMD), $\Delta$ SFI, $\Delta$ VFI, $\Delta$ TAI. SMI reduction between baseline and first evaluation was classified as early skeletal muscle mass depletion (ESMMD).

In addition, the quality of skeletal muscle at the time of the diagnosis was examined by calculating the mean attenuation (MA) of skeletal muscle and the intramuscular adipose tissue content (IMAC) of paraspinal muscles.

Therefore, only for baseline CT scans, MA, i.e., density of the skeletal muscle tissue was measured in $\mathrm{HU}$ and IMAC was calculated according to literature by dividing CT density of the multifidus muscles (HU) with CT density of subcutaneous fat (HU).

Higher IMAC indicates a greater content of adipose tissue in muscle and, consequently, suggests a lower skeletal muscle quality $[18,19]$.

We used specific cut-off values for SMI, MA, SFI and VFI. We used these cut-off values in accordance with their prognostic role highlighted in two large cohorts reported by Martin et al. and Ebady et al. [14,19]. Sarcopenia was defined as SMI $<43 \mathrm{~cm}^{2} / \mathrm{m}^{2}$ in male patients with BMI $<25 \mathrm{~kg} / \mathrm{m}^{2}$ and SMI $<53 \mathrm{~cm}^{2} / \mathrm{m}^{2}$ if BMI $>25 \mathrm{~kg} / \mathrm{m}^{2}$; in female patients, sarcopenia was set at SMI $<41 \mathrm{~kg} / \mathrm{m}^{2}$ irrespective of BMI. Cut-off values for MA were $<41 \mathrm{HU}$ in non-overweight patients $\left(\mathrm{BMI}<25 \mathrm{~kg} / \mathrm{m}^{2}\right.$ ) and $<33 \mathrm{HU}$ if BMI $>25 \mathrm{~kg} / \mathrm{m}^{2}$ for both sexes. Sarcopenic obesity was defined as sarcopenia combined with overweight or obesity (BMI $>25 \mathrm{~kg} / \mathrm{m}^{2}$ ). The cut off values for VFI, SFI and TAI were $52.9 \mathrm{~cm}^{2} / \mathrm{m}^{2}$ in males and $51.5 \mathrm{~cm}^{2} / \mathrm{m}^{2}$ in females, $50 \mathrm{~cm}^{2} / \mathrm{m}^{2}$ in males and $42 \mathrm{~cm}^{2} / \mathrm{m}^{2}$ in females and $107.7 \mathrm{~cm}^{2} / \mathrm{m}^{2}$ in males and $102.2 \mathrm{~cm}^{2} / \mathrm{m}^{2}$ in females, respectively [14,20].

\subsection{Statistical Analysis}

Data on baseline characteristics and body composition are shown as mean and SD. The median overall survival (OS) and progression free survival (PFS) were determined using the Kaplan-Meier method. Differences in demographic and clinical data between groups were evaluated using the Fisher exact test for categorical variables and independent $t$-test for continuous variables. The best cut-off for laboratory values were defined by ROC curve distribution.

Cox proportional hazards regression model was used to determine the relationship of explanatory variables to survival as hazard ratios (HR) and 95\% confidence intervals (CI). Logistic regression was used to describe and explain the relationship between dependent binary variables and independent variables. Odds ratio (OR) together with $95 \%$ confidence interval (CI) were provided for logistic regression analyses. Independent variable statistically significant in the univariate analyses were used to build the multivariate analysis. All tests were 2 -sided and $p<0.05$ was considered statistically significant.

MedCalc package (MedCalc1 version 16.8.4) was used for all statistical analyses.

\section{Results}

\subsection{Patients Characteristics}

The present study included 40 patients with a confirmed diagnosis of advanced gastric adenocarcinoma treated with first-line chemotherapy between November 2015 to December 2019 in Modena Cancer Center. The main characteristics of patients enrolled in the study are summarized in Table 1 . Overall, $82.5 \%$ of patients were younger than 70 years and the $60 \%$ of patients were male. The ECOG performance status was $0-1$ in the $87.5 \%$ of patients at baseline. Since we considered patients with advanced disease, in our sample, only six patients $(15 \%)$ were submitted to a previous gastrectomy. The first line regimens were mainly doublet chemotherapy with fluoropirimidin and platinum-derivative: 26 patients performed folfox (5-fluorouracil + oxaliplatin), four patients the TOGA regimen (cisplatin + 5-fluorouracil + trastuzumab), four patients a triplete regimen with EOX (epirubicin + oxaliplatin + capecitabine), three patients Xelox (capecitabine + oxaliplatin), and three patients monotherapy with 5-fluorouracil as De Gramont regimen. 
Table 1. Patients' characteristics.

\begin{tabular}{|c|c|}
\hline Variable & $\mathrm{N}(\%)$ \\
\hline \multicolumn{2}{|l|}{ Age } \\
\hline$<70$ years & $33(82.5 \%)$ \\
\hline$\geq 70$ years & $7(17.5 \%)$ \\
\hline \multicolumn{2}{|l|}{ Gender } \\
\hline Male & $24(60.0 \%)$ \\
\hline Female & $16(40.0 \%)$ \\
\hline \multicolumn{2}{|l|}{ Site of primary tumor } \\
\hline Gastroesophageal junction & $5(12.5 \%)$ \\
\hline Fundus & $3(7.5 \%)$ \\
\hline Body & $12(30.0 \%)$ \\
\hline Fundus and body & $4(10.0 \%)$ \\
\hline GE junction, fundus and body & $1(2.5 \%)$ \\
\hline Antrum & $7(17.5 \%)$ \\
\hline Body and antrum & $6(15.0 \%)$ \\
\hline Diffuse/linitis & $2(5.0 \%)$ \\
\hline \multicolumn{2}{|l|}{ Previous gastrectomy } \\
\hline Yes & $6(15.0 \%)$ \\
\hline No & $34(85 \%)$ \\
\hline \multicolumn{2}{|c|}{ Eastern Cooperative Oncology Group Performace Status (ECOG PS) at 1^line chemotherapy star } \\
\hline $0-1$ & $35(87.5 \%)$ \\
\hline$\geq 2$ & $5(12.5 \%)$ \\
\hline \multicolumn{2}{|l|}{$\mathrm{N}^{\circ}$ of metastatic sites at $1^{\wedge}$ line chemotherapy start } \\
\hline 1 & $12(30.0 \%)$ \\
\hline$\geq 2$ & $27(67.5 \%)$ \\
\hline Unknown & $1(2.5 \%)$ \\
\hline \multicolumn{2}{|l|}{ Metastatic site at $1^{\wedge}$ line chemotherapy start } \\
\hline Liver & $5(12.5 \%)$ \\
\hline Nodes & $11(27.5 \%)$ \\
\hline Peritoneum & $24(60.0 \%)$ \\
\hline Lung & $4(10.0 \%)$ \\
\hline Bone & $11(27.5 \%)$ \\
\hline Others & $5(12.5 \%)$ \\
\hline \multicolumn{2}{|l|}{ Body mass index-BMI $\left(\mathrm{kg} / \mathrm{m}^{2}\right)$} \\
\hline$<25$ & $28(70.0 \%)$ \\
\hline$\geq 25$ & $12(30.0 \%)$ \\
\hline \multicolumn{2}{|l|}{ Prognostic nutritional index-PNI } \\
\hline$<38.6$ & $8(20.0 \%)$ \\
\hline$\geq 38.6$ & $18(45.0 \%)$ \\
\hline Unknown & $14(35.0 \%)$ \\
\hline \multicolumn{2}{|l|}{ Type of first line treatment } \\
\hline Single agent & $2(5.0 \%)$ \\
\hline Combination & $38(95.0 \%)$ \\
\hline \multicolumn{2}{|l|}{ Laboratory parameter at first-line chemotherapy start } \\
\hline Neutrophil-lymphocyte Ratio-NLR (Mean \pm standard deviation) & $5.1 \pm 3.5$ \\
\hline$<4.8$ & $17(42.5 \%)$ \\
\hline$\geq 4.8$ & $11(27.5 \%)$ \\
\hline Unknown & $12(30.0 \%)$ \\
\hline Platelet-lymphocyte ratio-PLR (Mean \pm standard deviation) & $260.8 \pm 127.1$ \\
\hline$<217$ & $12(30.0 \%)$ \\
\hline$\geq 217$ & $16(40.0 \%)$ \\
\hline Unknown & $12(30.0 \%)$ \\
\hline
\end{tabular}


Table 1. Cont.

\begin{tabular}{|c|c|}
\hline Variable & $\mathbf{N}(\%)$ \\
\hline Lymphocyte-monocyte ratio-LMR (Mean \pm standard deviation) & $3.0 \pm 1.4$ \\
\hline$\leq 2.1$ & $7(17.5 \%)$ \\
\hline$>2.1$ & $21(52.5 \%)$ \\
\hline Unknown & $12(30.0 \%)$ \\
\hline $\begin{array}{c}\text { Systemic Immune-Inflammation Index }(\mathrm{SII})(\text { Mean } \pm \text { standard } \\
\text { deviation })\left(\times 10^{3} \text { cells } / \mu \mathrm{L}\right.\end{array}$ & $1465 \pm 1041$ \\
\hline$\leq 1110$ & $20(30.0 \%)$ \\
\hline$>1110$ & $16(40.0 \%)$ \\
\hline Unknown & $12(30.0 \%)$ \\
\hline Albumine (Mean \pm standard deviation) $(\mathrm{g} / \mathrm{dL})$ & $3.6 \pm 0.5$ \\
\hline$\leq 3.5$ & $19(47.5 \%)$ \\
\hline$>3.5$ & $17(42.5 \%)$ \\
\hline Unknown & $4(10.0 \%)$ \\
\hline $\begin{array}{c}\mathrm{C} \text { reactive Protein-PCR (Mean } \pm \text { standard deviation }) \\
(\mathrm{mg} / \mathrm{dL})\end{array}$ & $3,2 \pm 4,6$ \\
\hline$<2.2$ & $13(32.5 \%)$ \\
\hline$\geq 2.2$ & $8(20.0 \%)$ \\
\hline Unknown & $19(47.5 \%)$ \\
\hline $\begin{array}{c}\text { Carcinoembryonic antigen-CEA (Mean } \pm \text { standard deviation) } \\
(\mathrm{ng} / \mathrm{mL})\end{array}$ & $201.6 \pm 683.2$ \\
\hline 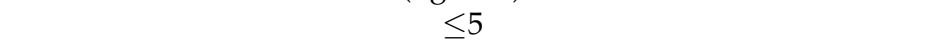 & $23(57.5 \%)$ \\
\hline$>5$ & $14(35.0 \%)$ \\
\hline Unknown & $3(7.5 \%)$ \\
\hline $\begin{array}{c}\text { Carbohydrate antigen } 19.9-\text { Ca } 19.9 \text { (Mean } \pm \text { standard deviation) } \\
(\mathrm{U} / \mathrm{mL})\end{array}$ & $622 \pm 1856$ \\
\hline$\leq 37$ & $20(50.0 \%)$ \\
\hline$>37$ & $17(42.5 \%)$ \\
\hline Unknown & $3(7.5 \%)$ \\
\hline
\end{tabular}

The median duration of first line chemotherapy was 6 months (range 1.68-16.38 months); in 7 of $40(17 \%)$ patients an early discontinuation of the treatment was required due to toxicities or worsening of patient clinical conditions.

The mainly reported toxicity was blood count alteration with neutropenia and anemia in 11 patients (27\%); afterward, gastrointestinal alterations (mainly nausea and vomit) and peripheral neuropathy in 8 patients $(19 \%)$. The grade of these adverse events was not reported in our clinical records but none of these was a grade 4 . Overall, 20 patients $(50 \%)$ were reported to receive a second line therapy, consisting prevalently in the association ramucirumab and paclitaxel accordi2ng to the guidelines. In particular, $8 / 20(40 \%)$ patients in the first group and $12 / 20(60 \%)$ patients in second group were treated with a second line therapy.

Concerning the anthropometric characteristics, median BMI of the entire sample was $23.59 \mathrm{~kg} / \mathrm{m}^{2}$. The prevalence of baseline sarcopenia and sarcopenic obesity were $42.5 \%$ $(17 / 40)$ and $7 \%(3 / 40)$, respectively.

\subsection{Prognostic Factors}

The first part of our analysis was addressed to research clinical and anthropometric prognostic parameters and the correlation between these variables and clinical benefit in the whole sample.

Overall, after a median follow-up of 16.4 months, mOS was 12.07 months, whereas mPFS was 6.18 months.

All covariates retrieved were tested within a univariate model.

We evaluated the prognostic impact of baseline clinical, laboratory and anthropometric measures finding a significant interaction between ECOG (ECOG 2 vs. ECOG 0/1. HR 
12.74; 95\% C.I. 0.66 to 243.85, $p<0.001$ ), LMR (LMR > 2.1 vs. $\mathrm{LMR}<2.1$. HR 3.47; 95\% C.I. 1.35 to $8.91, p$ 0.0095), PCR (PCR > 2.2 vs. PCR < 2.2. HR 3.71; 95\% C.I. 1.38 to 9.93, $p$ 0.009), PNI (PNI > 38.6 vs. PNI < 38.6. HR 3.58; 95\% C.I. 1.36 to 9.42, $p 0.009$ ) and overall survival. Concerning the anthropometric parameters, only ESMMD $>10 \%$ from baseline to the first radiological revaluation significantly impact on mOS (HR 2.57, 95\% CI 1.13-5.82, $p=0.0023$ ) (Table 2).

Following adjustment for significantly prognostic covariates at univariate analysis, a multivariate analysis was performed, which confirmed ECOG PS (0-1 vs. 2 HR 49.32, 95\% CI 7.32-331.95, $p=0.0001)$ and ESMMD > 10\% (HR 2.47 95\% CI 1.05-7.09, $p=0.0375)$ as the only independent prognostic factors in terms of OS and PFS (Figure 2).

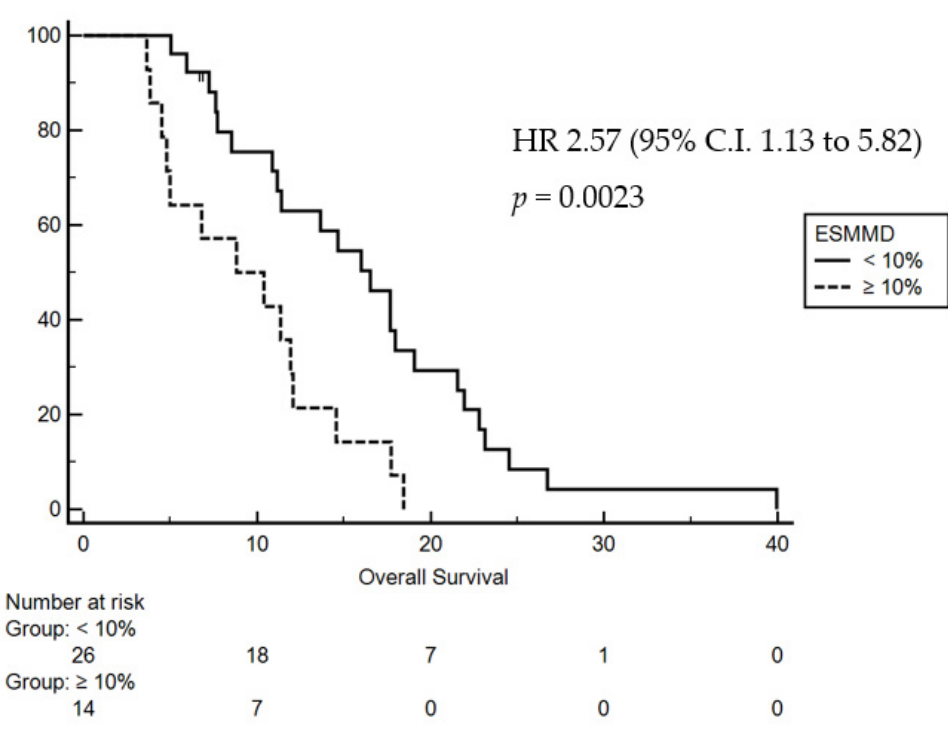

(a)

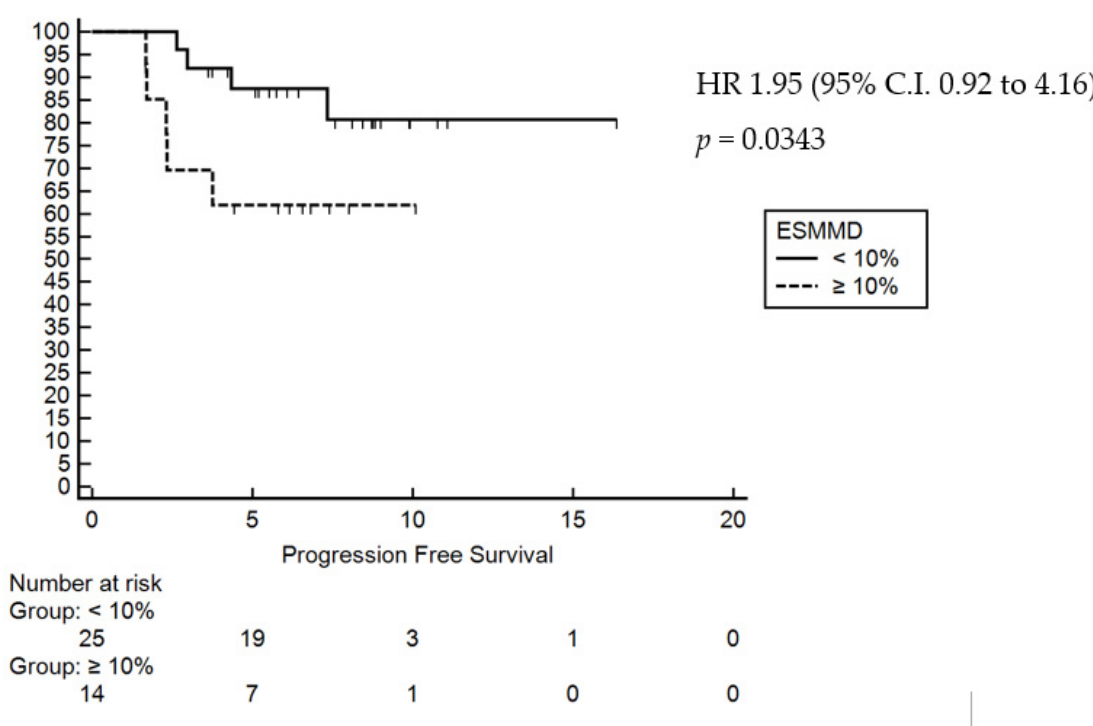

(b)

Figure 2. Kaplan-Meier curves for overall survival (a) and progression free survival (b) according to early skeletal muscle mass depletion (ESMMD). HR: Hazard Ration; CI: Confidence Interval.

Moreover, by performing a logistic regression analysis, ECOG PS was highlighted to be the only clinical parameter correlated with clinical benefit (defined as stable disease and/or partial response vs. progression disease) (OR 7.25, 95\% C.I. 0.9876 to 53.2239, $p=0.0004)$.

Overall, no correlation has been highlighted between anthropometric parameters and toxicities from treatment. 
On the other hand, a relationship has been reported between several clinical and bio-humoral variables and radiological assessment of early variation of SMI. In particular, our analysis confirmed a strong link between ESMMD and baseline LDH > $460 \mathrm{U} / 1$ (OR 7.91; 95\% CI 1.31-47.51, $p=0.0046$ ), baseline CRP > 2.2 mg/dL (OR 20.0; 95\% CI 1.65-241.73, $p=0.006$ ) and weight decrease during treatment (OR 0.82; 95\% C.I. 0.71 to $0.94, p=0.0009$ ).

Table 2. Uni and multivariate analysis for overall survival.

\begin{tabular}{|c|c|c|c|}
\hline & \multicolumn{2}{|l|}{ Univariate } & \multirow[t]{2}{*}{ Multivariate } \\
\hline & $\operatorname{HR}(95 \% \mathrm{CI})$ & $p$ & \\
\hline Age ( $\geq 65$ vs. $<65$ years) & $1.08(0.57-2.07)$ & 0.8110 & \\
\hline ECOG PS (2 vs. $0-1)$ & $12.75(0.67-243.86)$ & $<0.0001$ & 0.0001 \\
\hline Site of $M(>1$ vs. 1$)$ & $1.92(0.90-4.11)$ & 0.058 & \\
\hline $\operatorname{NLR}(\geq 4.8$ vs. $<4.8)$ & $2.00(0.84-4.75)$ & 0.0711 & \\
\hline $\operatorname{LMR}(<2.1$ vs. $\geq 2.1)$ & $3.03(0.88-10.46)$ & 0.0060 & \\
\hline $\operatorname{PCR}(\geq 2.2$ vs. $<2.2 \mathrm{mg} / \mathrm{dL})$ & $3.1(0.98-9.78)$ & 0.0055 & \\
\hline $\mathrm{CEA}(\geq 5$ vs. $<5 \mathrm{ng} / \mathrm{mL})$ & $0.67(0.33$ to 1.36$)$ & 0.2763 & \\
\hline PNI $(\geq 38.6$ vs. $<38.6)$ & $0.34(0.11-1.06)$ & 0.0058 & \\
\hline SII $\left(\geq 1110\right.$ vs. $<1110\left(\times 10^{3}\right.$ cells $\left./ \mu \mathrm{L}\right)$ & $0.95(0.44-2.07)$ & 0.9104 & \\
\hline BMI $\left(<25\right.$ vs. $\left.\geq 25 \mathrm{~kg} / \mathrm{m}^{2}\right)$ & $0.79(0-37-1.70)$ & 0.5215 & \\
\hline IMAC ( $\leq-0.33$ vs. $>-0.33)$ & $0.67(0.34-1.32)$ & 0.2233 & \\
\hline $\begin{array}{c}\text { VFI }\left(\geq 52.9 \mathrm{~cm}^{2} / \mathrm{m}^{2} \text { in males and }\right. \\
51.5 \mathrm{~cm}^{2} / \mathrm{m}^{2} \text { in females vs. }<52.9 \mathrm{~cm}^{2} / \mathrm{m}^{2} \\
\left.\text { in males and } 51.5 \mathrm{~cm}^{2} / \mathrm{m}^{2} \text { in females }\right)\end{array}$ & $1.24(0.66-2.36)$ & 0.5010 & \\
\hline $\begin{array}{l}\text { SFI }\left(\leq 50 \mathrm{~cm}^{2} / \mathrm{m}^{2} \text { in males and } 42 \mathrm{~cm}^{2} / \mathrm{m}^{2}\right. \\
\text { in females vs. }>50 \mathrm{~cm}^{2} / \mathrm{m}^{2} \text { in males and } 42 \\
\left.\mathrm{~cm}^{2} / \mathrm{m}^{2} \text { in females }\right)\end{array}$ & $0.76(0.40-1.43)$ & 0.3921 & \\
\hline Sarcopenia sec. Martin (Yes vs. No) & $1.40(0.72-2.73)$ & 0.3058 & \\
\hline $\begin{array}{l}\text { Sarcopenia at revaluation sec. Martin (Yes } \\
\text { vs. No) }\end{array}$ & $2.24(1.07-4.69$ & 0.0117 & \\
\hline ESMMD ( $\geq 10 \%$ vs. $<10 \%)$ & $2.57(1.13-5.83)$ & 0.0036 & 0.0375 \\
\hline
\end{tabular}

\subsection{Role of Nutritional Assessment}

The second part of our analysis was focused on the role of an early and scheduled nutritional evaluation. We searched for differences in terms of clinical, anthropometric and survival outcomes between the first group of patients which received a nutritional evaluation at oncology's discretion and the second group of patients which received a standardized nutritional evaluation at the baseline and then every 2-4 weeks during treatment. Overall, 20 patients were included in the first group of patients and 20 patients were included in the second group of patients.

Clinical characteristics were well balanced between the two groups of patients as shown in Table 3.

No significant differences in terms of OS and PFS were reported between the two groups of patients; in return, significative differences in adipose tissue modification during treatment have been highlighted. In particular, our analysis showed that patients reserved to a nutritional scheduled support experienced a mean gain in subcutaneous fat (SFI) of $11.4 \%$ at the first radiological evaluation vs. baseline; contrarily, patients reserved to an occasional nutritional support experienced a mean lost in SFI of $3.97 \%$ at the first radiological evaluation vs. baseline. Consistently, we demonstrated in patients submitted to a scheduled nutritional support a mean gain in visceral fat of $8.55 \%$ at the first radiological evaluation vs. baseline, whereas in patients without a scheduled nutritional evaluation, we found a mean loss of visceral fat of $10.21 \%$ at the first revaluation vs. baseline. No significant differences in median ESMMD $(-7.52 \%$ vs. $-2.94 \%)$ or median $\triangle \mathrm{BMI}(-3.7 \%$ vs. $-1.7 \%$ ) were reported in the two groups of patients. 
Table 3. Patients categorized according to the nutritional approach.

\begin{tabular}{|c|c|c|c|}
\hline & $\begin{array}{c}\text { Occasional } \\
\text { Nutritional Evaluation } \\
\qquad(n=20)\end{array}$ & $\begin{array}{c}\text { Systematic Nutritional } \\
\text { Evaluation } \\
(n=20)\end{array}$ & $p$ \\
\hline \multicolumn{4}{|c|}{ Age } \\
\hline$<70$ years & $16(80 \%)$ & $17(85 \%)$ & 1.00 \\
\hline$\geq 70$ years & $4(20 \%)$ & $3(15 \%)$ & \\
\hline \multicolumn{4}{|c|}{ Gender } \\
\hline Male & $13(65 \%)$ & $11(55 \%)$ & 0.54 \\
\hline Female & $7(35 \%)$ & $9(45 \%)$ & \\
\hline \multicolumn{4}{|c|}{ Site of primary tumor } \\
\hline Gastroesophageal junction & $4(20 \%)$ & $1(5 \%)$ & 0.2334 \\
\hline Fundus & $3(15 \%)$ & 0 & \\
\hline Body & $4(20 \%)$ & $8(40 \%)$ & \\
\hline Fundus and body & $2(10 \%)$ & $2(10 \%)$ & \\
\hline GE junction, fundus and body & $1(5 \%)$ & 0 & \\
\hline Antrum & $3(15 \%)$ & $4(20 \%)$ & \\
\hline Body and antrum & $3(15 \%)$ & $3(15 \%)$ & \\
\hline Diffuse/linitis & 0 & $2(10 \%)$ & \\
\hline \multicolumn{4}{|c|}{ Previous gastrectomy } \\
\hline Yes & $3(15 \%)$ & $3(15 \%)$ & \\
\hline No & $17(85 \%)$ & $17(85 \%)$ & \\
\hline \multicolumn{4}{|c|}{ ECOG PS at 1^line chemotherapy start } \\
\hline $0-1$ & $19(95 \%)$ & $16(80 \%)$ & 0.3416 \\
\hline$\geq 2$ & $1(5 \%)$ & $4(20 \%)$ & \\
\hline \multicolumn{4}{|c|}{$\begin{array}{c}\mathrm{N}^{\circ} \text { of metastatic sites at } 11^{\wedge} \text { line } \\
\text { chemotherapy start }\end{array}$} \\
\hline 1 & $7(35 \%)$ & $5(25 \%)$ & 0.7311 \\
\hline$\geq 2$ & $13(65 \%)$ & $14(70 \%)$ & \\
\hline Unknown & 0 & $1(5 \%)$ & \\
\hline \multicolumn{4}{|c|}{$\begin{array}{l}\text { Metastatic site at } 1 \wedge \text { line } \\
\text { chemotherapy start }\end{array}$} \\
\hline Liver & $1(5 \%)$ & $4(20 \%)$ & 0.1173 \\
\hline Nodes & $8(40 \%)$ & $3(15 \%)$ & \\
\hline Peritoneum & $11(55 \%)$ & $13(65 \%)$ & \\
\hline Lung & $1(5 \%)$ & $3(15 \%)$ & \\
\hline Bone & $8(40 \%)$ & $3(15 \%)$ & \\
\hline Others & $4(20 \%)$ & $1(5 \%)$ & \\
\hline \multicolumn{4}{|c|}{ Body mass index-BMI $\left(\mathrm{kg} / \mathrm{m}^{2}\right)$} \\
\hline$<25$ & $14(70 \%)$ & $14(70 \%)$ & 1.00 \\
\hline$\geq 25$ & $6(30 \%)$ & $6(30 \%)$ & \\
\hline \multicolumn{4}{|c|}{ Prognostic nutritional index-PNI } \\
\hline$<38.6$ & $2(10 \%)$ & $6(30 \%)$ & 0.2077 \\
\hline$\geq 38.6$ & $9(45 \%)$ & $9(45 \%)$ & \\
\hline Unknown & $9(45 \%)$ & $5(25 \%)$ & \\
\hline \multicolumn{4}{|c|}{ Type of first line treatment } \\
\hline Single agent & $1(5 \%)$ & $1(5 \%)$ & 1.00 \\
\hline Combination & $19(95 \%)$ & $19(95 \%)$ & \\
\hline \multicolumn{4}{|c|}{$\begin{array}{l}\text { Laboratory parameter at first-line } \\
\text { chemotherapy start }\end{array}$} \\
\hline $\begin{array}{l}\text { Neutrophil-lymphocyte Ratio- } \\
\text { NLR (Mean } \pm \text { standard deviation) }\end{array}$ & $4.6 \pm 1.9$ & $5.5 \pm 4.2$ & \\
\hline$<4.8$ & $7(35 \%)$ & $10(50 \%)$ & 0.3765 \\
\hline$\geq 4.8$ & $5(25 \%)$ & $6(30 \%)$ & \\
\hline Unknown & $8(40 \%)$ & $4(20 \%)$ & \\
\hline
\end{tabular}


Table 3. Cont.

\begin{tabular}{|c|c|c|c|}
\hline & $\begin{array}{c}\text { Occasional } \\
\text { Nutritional Evaluation } \\
\quad(n=20)\end{array}$ & $\begin{array}{c}\text { Systematic Nutritional } \\
\text { Evaluation } \\
(n=20)\end{array}$ & $p$ \\
\hline $\begin{array}{c}\text { Platelet-lymphocyte ratio-- } \\
\text { PLR (Mean } \pm \text { standard deviation) }\end{array}$ & $240.4 \pm 81.9$ & $276.1 \pm 150.6$ & \\
\hline$<217$ & $5(25 \%)$ & $7(35 \%)$ & 0.3835 \\
\hline$\geq 217$ & $7(35 \%)$ & $9(45 \%)$ & \\
\hline Unknown & $8(40 \%)$ & $4(20 \%)$ & \\
\hline $\begin{array}{c}\text { Lymphocyte-monocyte ratio- } \\
\text { LMR (Mean } \pm \text { standard deviation) }\end{array}$ & $2.8 \pm 1.4$ & $3.1 \pm 1.5$ & \\
\hline$\leq 2.1$ & $3(15 \%)$ & $4(20 \%)$ & 0.3858 \\
\hline$>2.1$ & $9(45 \%)$ & $12(60 \%)$ & \\
\hline Unknown & $8(40 \%)$ & $4(20 \%)$ & \\
\hline $\begin{array}{c}\text { Lactate dehydrogenase- } \\
\text { LDH (Mean } \pm \text { standard deviation) }(\mathrm{U} / \mathrm{L})\end{array}$ & $631 \pm 460$ & $505 \pm 661$ & \\
\hline$\leq 460$ & $8(40 \%)$ & $15(75 \%)$ & 0.0769 \\
\hline$>460$ & $6(30 \%)$ & $2(10 \%)$ & \\
\hline Unknown & $6(30 \%)$ & $3(15 \%)$ & \\
\hline Albumine (Mean \pm standard deviation) (g/dL) & $3,7 \pm 0,5$ & $3,4 \pm 0,5$ & \\
\hline$\leq 3.5$ & $8(40 \%)$ & $11(55 \%)$ & 0.6056 \\
\hline$>3.5$ & $10(50 \%)$ & $7(35 \%)$ & \\
\hline Unknown & $2(10 \%)$ & $2(10 \%)$ & \\
\hline $\begin{array}{c}\text { C reactive Protein- } \\
\text { PCR (Mean } \pm \text { standard deviation) }(\mathrm{mg} / \mathrm{dL})\end{array}$ & $3.7 \pm 5.2$ & $3.0 \pm 4.2$ & \\
\hline$<2.2$ & $4(20 \%)$ & $9(45 \%)$ & 0.0820 \\
\hline$\geq 2.2$ & $3(15 \%)$ & $5(25 \%)$ & \\
\hline Unknown & $13(65 \%)$ & $6(30 \%)$ & \\
\hline $\begin{array}{c}\text { Carcinoembryonic antigen- } \\
\text { CEA (Mean } \pm \text { standard deviation) }(\mathrm{ng} / \mathrm{mL})\end{array}$ & $178.5 \pm 537.3$ & $216.2 \pm 744.4$ & \\
\hline$\leq 5$ & $11(55 \%)$ & $12(60 \%)$ & 0.8283 \\
\hline$>5$ & $7(35 \%)$ & $7(35 \%)$ & \\
\hline Unknown & $2(10 \%)$ & $1(5 \%)$ & \\
\hline $\begin{array}{c}\text { Carbohydrate antigen } 19.9- \\
\text { Ca } 19.9 \text { (Mean } \pm \text { standard deviation })(\mathrm{U} / \mathrm{mL})\end{array}$ & $646.7 \pm 1013.6$ & $604.9 \pm 2217.4$ & \\
\hline$\leq 37$ & $9(45 \%)$ & $11(55 \%)$ & 0.529 \\
\hline$>37$ & $9(45 \%)$ & $8(40 \%)$ & \\
\hline Unknown & $2(10 \%)$ & $1(5 \%)$ & \\
\hline
\end{tabular}

\section{Discussion}

Recently, the role of sarcopenia in gastric cancer has been focused among perioperative setting. Preoperative muscle mass quality and malnutrition are strictly related to higher surgical risk and delayed and prematurely interrupted adjuvant treatment [21]. Few data are available on the prognostic role of malnutrition in mGC patients [22,23].

In this study, we evaluated a small cohort of 40 advanced GC patients treated with first line palliative chemotherapy in Modena Cancer Center between 2016 and 2019. We evaluated the prognostic impact of baseline clinical, laboratory and anthropometric measures finding a significant interaction between ECOG (ECOG 0/1 vs. ECOG 2, $p<0.001$ ), LMR (LMR $>2.1$ vs. LMR $<2.1, p 0.0095)$, PCR (PCR $>2.2$ vs. $\mathrm{PCR}<2.2, p 0.009$ ), PNI (PNI $>38.6$ vs. $\mathrm{PNI}<38.6, p 0.009)$ and overall survival.

In our group of patients, the prevalence of baseline sarcopenia and sarcopenic obesity were $42.5 \%(17 / 40)$ and $7 \%(3 / 40)$ but neither baseline sarcopenia nor BMI at diagnosis significantly affected survival. Conversely, ESMMD > 10\% (reported in 14/40 patients) and sarcopenia at first re-evaluation (reported in 18/40 patients) during palliative chemotherapy were associated with shorter $\mathrm{mPFS}$ and mOS. In multivariate analysis, in addition to ECOG performance status, only early SMI reduction $>10 \%$ was statistically relevant and associated with both worse PFS and OS (median PFS 7.3 months vs. 4.4 months, $p$ 0.038; median OS 
16.5 months vs. 8.5 months, $p$ 0.0375). The relevant role of ECOG performance status was highlighted also for its significant impact on clinical benefit at first evaluation (OR 7.25; 95\% C.I. 0.9876 to 53.2239; $p$ 0.049).

The prevailing role of ESMMD over baseline sarcopenia in metastatic setting has been previously reported not only for gastric cancer patients [20] but also in patients with different gastrointestinal cancers [24-27]. In the IMPACT trial, in particular, in patients with metastatic pancreatic cancer, ESMMD $\geq 10 \%$ was significantly associated with worse OS (HR: 2.16; 95\% CI 1.23-3.78; $p=0.007$ ) and PFS (HR: 2.31; 95\% CI 1.30-4.09; $p=0.004$ ) [27].

Beside the role of SMI depletion, another point of our study was the evaluation of the prognostic impact of muscle tissue quality (IMAC and MA) as well as early changes in subcutaneous and visceral adipose tissue during chemotherapy, but none of these parameters were clinically relevant maybe due to the small sample size of the study. The prognostic role of muscle quality at baseline is reported in other setting [19,27-31] but in a recent Dutch series enrolled $88 \mathrm{mGC}$ patients treated with capecitabine and oxaliplatin as first line chemotherapy, no correlation between muscle attenuation and survival was confirmed [24].

Several studies confirmed a well-known association between muscle mass depletion and systemic inflammation $[21,32,33]$. Systemic inflammation has been reported as a strong prognostic factor in cancer progression. In patients with cancer, pro-inflammatory mediators cause an energetic imbalance between catabolic and anabolic pathways. IL-6, IL-2, IL-10, epidermal growth factor (EGF) and IFN exert their effect by activating the signal transducer and activator of transcription 3 (STAT3) producing loss of muscle mass. The levels of IL-6, TNFa and CRP have been reported to be significantly up-regulated in sarcopenia patients. The activation of a pro-inflammatory status can also lead to insulin resistance and muscle depletion through the activation of the ubiquitin-proteasome proteolytic pathway [34]. In our series, we confirmed the strong link between early muscle mass loss and systemic inflammatory indexes because we noted that baseline LDH > 460, CRP > 2.2 and weight decrease during treatment significantly predicted ESMMD.

The second part of our work was an exploratory analysis on the role of a scheduled nutritional evaluation.

As we know, the nutritional support should be considered as an integrated treatment in patients receiving palliative anti-cancer treatment. Patients identified by screening for unintentional weight loss in last 3-6 years or decreased oral intake, should receive adequate nutritional counselling and support but few studies reported a significative benefit of nutritional support in advanced cancer patients [12,17,35,36].

In our center from 2018, all patients with mGC were evaluated through MUST screening test and then taking charge from a nutritional perspective. They performed a nutritional evaluation every 2-4 weeks alongside the scheduled oncological examination. Before 2018, the nutritional path was at the discretion of oncologists and often the first nutritional evaluation was delayed.

Twenty patients were diagnosed in 2016-2017 and subjected to an occasional involvement of the nutritional team; conversely, the other group of patients were evaluated at diagnosis and then every 2 or 4 weeks with scheduled nutritional visit. No significant difference in OS and PFS were reported but relevant difference in adipose tissue modification has been found.

About subcutaneous fat, a mean gain of $15.38 \%$ of SFI vs. baseline SFI was reported between patients with nutritional scheduled support $(+11.4 \%$ at 3 months vs. baseline) vs. patients with occasional nutritional evaluation $(-3.97 \%$ at 3 months vs. baseline). Concurrently, a relevant mean gain of $18.76 \%$ was reported about the visceral fat between patients in the first group $(+8.55 \%$ vs. baseline) vs. patients in the latter one $(-10.21 \%$ vs. baseline).

No relevant difference in median ESMMD $(-7.52 \%$ vs. $-2.94 \%)$ or median $\triangle \mathrm{BMI}$ $(-3.7 \%$ vs. $-1.7 \%)$ were reported between the 2 groups. 
The prognostic role of adipose tissue in gastrointestinal cancers is still debating [37]. In patients with pancreatic and biliary carcinoma underwent resection, high VSR (visceral to subcutaneous ratio) was reported as a negative prognostic factor in a single Japanese series [38,39]. In a classic trial, enrolled patients underwent gastrectomy followed by adjuvant chemotherapy, a marked loss of visceral or subcutaneous fat significantly predicted shorter DFS and OS [40].

In a large series of 1473 gastrointestinal and respiratory metastatic cancer patients, low TAI (total adipose index) was associated with increased mortality (mOS 19.8 months vs. 14.0 months) [19]. In metastatic colorectal cancer, higher VFI has been associated with shorter OS in patients treated with chemotherapy and anti-VEGF antibody but not in those treated with chemotherapy alone [41]. In a post hoc analysis of two non-randomized phase II trials in the same setting, low SFI (HR 1.63; 1.23-2.17) and low VFI (HR 1.48; 1.09-2.02) were associated with an increased risk of dying confirming the protective role of obesity [42].

In addition, in our series, this protective role was confirmed although not statistically significative maybe due to the small sample size: patients with a higher baseline SFI $\left(>50 \mathrm{~cm}^{2} / \mathrm{m}^{2}\right.$ in males and $>42 \mathrm{~cm}^{2} / \mathrm{m}^{2}$ in females) experienced an increased median overall survival (13.65 vs. 11.94 months), such as patients with a higher VFI $\left(>52.9 \mathrm{~cm}^{2} / \mathrm{m}^{2}\right.$ in males and $>51.5 \mathrm{~cm}^{2} / \mathrm{m}^{2}$ in females; mOS 13.65 vs. 11.34 months). Moreover, patients with VFI gain during chemotherapy experienced an increased median overall survival (16.02 months vs. 11.18 months), such as patients with SFI gain (14.64 months vs. 11.18 months) and patients with TAI gain (13.65 months vs. 11.35 months).

Our analysis has several limitations: firstly, the limited number of patients and the retrospective design of the trial. Secondly, we reported a number of patients treated with a consequent second line therapy, which could have influenced the survival outcomes of the two groups of patients considered. Thirdly, patients without available CT scans or lost at follow up were excluded from this trial leading to a possible selection bias. Moreover, we could not have a comprehensive report of the relation between body composition parameters and chemotherapy toxicities or quality of life due to few medical records about these items and the retrospective nature of the study.

\section{Conclusions}

In conclusion, as reported in other metastatic setting, we confirm the prognostic impact of ESMMD > 10\% during the first 3 months of first line chemotherapy in metastatic gastric cancer. The impact of ESMMD $>10 \%$ is independent from weight loss and could be predicted by some immune-inflammatory markers such as CRP and LDH at baseline.

The prognostic role of a scheduled nutritional assessment could not be highlighted, perhaps due to the sample size of this series, but a relevant gain in adipose tissue (SFI, VFI, TAI) is reported in this group of patients, suggesting clinical benefit due to the protective role of obesity in metastatic gastrointestinal cancers; therefore, a scheduled nutritional assessment and intervention should be evaluated in metastatic gastric cancer patients. However, the role of this approach deserves further confirmation in large prospective trials.

Author Contributions: Conception and design: A.S., M.S., A.P., F.P. (Francesco Prampolini), C.B., F.V.; Acquisition of data (acquired and managed patients): All authors; Analysis and interpretation of data: A.S., F.P. (Francesco Prampolini); Writing, review and/or revision of the manuscript: A.S., M.R., F.C., F.P. (Francesco Prampolini); Final approval of manuscript: All authors. All authors have read and agreed to the published version of the manuscript.

Funding: This research received no external funding.

Institutional Review Board Statement: The Ethical Review Board of each Institutional Hospital approved the present study. This study was performed in line with the principles of the Declaration of Helsinki.

Informed Consent Statement: Informed consent was obtained from all subjects involved in the study. 
Data Availability Statement: Data available on request from the authors.

Conflicts of Interest: The authors declare no conflict of interest.

\section{References}

1. Salati, M.; Di Emidio, K.; Tarantino, V.; Cascinu, S. Second-line treatments: Moving towards an opportunity to improve survival in advanced gastric cancer? ESMO Open 2017, 2, e000206. [CrossRef]

2. Wagner, A.D.; Syn, N.L.X.; Moehler, M.; Grothe, W.; Yong, W.P.; Tai, B.-C.C.; Ho, J.; Unverzagt, S.; Wagner, D.A.; Syn, L.X.N.; et al. Chemotherapy for advanced gastric cancer Systematic Review. Cochrane Database Syst. Rev. 2017, 2017, 8. [CrossRef]

3. Shitara, K.; Bang, Y.-J.; Iwasa, S.; Sugimoto, N.; Ryu, M.-H.; Sakai, D.; Chung, H.-C.; Kawakami, H.; Yabusaki, H.; Lee, J.; et al. Trastuzumab Deruxtecan in Previously Treated HER2-Positive Gastric Cancer. N. Engl. J. Med. 2020, 382, 2419-2430. [CrossRef]

4. Shitara, K.; Van Cutsem, E.; Bang, Y.J.; Fuchs, C.; Wyrwicz, L.; Lee, K.W.; Kudaba, I.; Garrido, M.; Chung, H.C.; Lee, J.; et al. Efficacy and Safety of Pembrolizumab or Pembrolizumab Plus Chemotherapy vs Chemotherapy Alone for Patients with First-line, Advanced Gastric Cancer: The KEYNOTE-062 Phase 3 Randomized Clinical Trial. JAMA Oncol. 2020, 6, 1571-1580. [CrossRef] [PubMed]

5. Wilke, H.; Muro, K.; Van Cutsem, E.; Oh, S.C.; Bodoky, G.; Shimada, Y.; Hironaka, S.; Sugimoto, N.; Lipatov, O.; Kim, T.Y.; et al. Ramucirumab plus paclitaxel versus placebo plus paclitaxel in patients with previously treated advanced gastric or gastro-oesophageal junction adenocarcinoma (RAINBOW): A double-blind, randomised phase 3 trial. Lancet Oncol. 2014, 15, 1224-1235. [CrossRef]

6. Fuchs, C.S.; Tomasek, J.; Yong, C.J.; Dumitru, F.; Passalacqua, R.; Goswami, C.; Safran, H.; dos Santos, L.V.; Aprile, G.; Ferry, D.R.; et al. Ramucirumab monotherapy for previously treated advanced gastric or gastro-oesophageal junction adenocarcinoma (REGARD): An international, randomised, multicentre, placebo-controlled, phase 3 trial. Lancet 2014, 383, 31-39. [CrossRef]

7. Bang, Y.-J.; Van Cutsem, E.; Feyereislova, A.; Chung, H.C.; Shen, L.; Sawaki, A.; Lordick, F.; Ohtsu, A.; Omuro, Y.; Satoh, T.; et al. Trastuzumab in combination with chemotherapy versus chemotherapy alone for treatment of HER2-positive advanced gastric or gastro-oesophageal junction cancer (ToGA): A phase 3, open-label, randomised controlled trial. Lancet 2010, 376, 687-697. [CrossRef]

8. Demirelli, B.; Babacan, N.A.; Ercelep, Ö.; Öztürk, M.A.; Kaya, S.; Tanrıkulu, E.; Khalil, S.; Hasanov, R.; Alan, Ö.; Telli, T.A.; et al. Modified Glasgow Prognostic Score, Prognostic Nutritional Index and ECOG Performance Score Predicts Survival Better than Sarcopenia, Cachexia and Some Inflammatory Indices in Metastatic Gastric Cancer. Nutr. Cancer 2021, 73, 230-238. [CrossRef]

9. Ter Veer, E.; van Kleef, J.J.; Schokker, S.; van der Woude, S.O.; Laarman, M.; Mohammad, N.H.; Sprangers, M.A.; van Oijen, M.G.; van Laarhoven, H.W. Prognostic and predictive factors for overall survival in metastatic oesophagogastric cancer: A systematic review and meta-analysis. Eur. J. Cancer 2018, 103, 214-226. [CrossRef]

10. Casadei-Gardini, A.; Scarpi, E.; Ulivi, P.; Palladino, M.A.; Accettura, C.; Bernardini, I.; Spallanzani, A.; Gelsomino, F.; Corbelli, J.; Marisi, G.; et al. Prognostic role of a new inflammatory index with neutrophil-to-lymphocyte ratio and lactate dehydrogenase (CII: Colon Inflammatory Index) in patients with metastatic colorectal cancer: Results from the randomized Italian Trial in Advanced Colorectal Cancer. Cancer Manag. Res. 2019, 11, 4357-4369. [CrossRef]

11. Grenader, T.; Waddell, T.; Peckitt, C.; Oates, J.; Starling, N.; Cunningham, D.; Bridgewater, J. Prognostic value of neutrophil-tolymphocyte ratio in advanced oesophago-gastric cancer: Exploratory analysis of the REAL-2 trial. Ann. Oncol. 2016, 27, 687-692. [CrossRef]

12. Arends, J.; Bachmann, P.; Baracos, V.; Barthelemy, N.; Bertz, H.; Bozzetti, F.; Fearon, K.; Hütterer, E.; Isenring, E.; Kaasa, S.; et al. ESPEN guidelines on nutrition in cancer patients. Clin. Nutr. 2017, 36, 11-48. [CrossRef]

13. Cushen, S.J.; Power, D.G.; Murphy, K.P.; McDermott, R.; Griffin, B.T.; Lim, M.; Daly, L.; MacEneaney, P.; O’Sullivan, K.; Prado, C.M.; et al. Impact of body composition parameters on clinical outcomes in patients with metastatic castrate-resistant prostate cancer treated with docetaxel. Clin. Nutr. ESPEN 2016, 13, e39-e45. [CrossRef]

14. Martin, L.; Birdsell, L.; MacDonald, N.; Reiman, T.; Clandinin, M.T.; McCargar, L.J.; Murphy, R.; Ghosh, S.; Sawyer, M.B.; Baracos, V.E. Cancer Cachexia in the Age of Obesity: Skeletal Muscle Depletion Is a Powerful Prognostic Factor, Independent of Body Mass Index. J. Clin. Oncol. 2013, 31, 1539-1547. [CrossRef]

15. Ryan, A.M.; Prado, C.M.; Sullivan, E.S.; Power, D.G.; Daly, L.E. Effects of weight loss and sarcopenia on response to chemotherapy, quality of life, and survival. Nutrition 2019, 67-68, 110539. [CrossRef]

16. Omarini, C.; Palumbo, P.; Pecchi, A.; Draisci, S.; Balduzzi, S.; Nasso, C.; Barbolini, M.; Isca, C.; Bocconi, A.; Moscetti, L.; et al. Predictive Role of Body Composition Parameters in Operable Breast Cancer Patients Treated with Neoadjuvant Chemotherapy. Cancer Manag. Res. 2019, 11, 9563-9569, PMCID:PMC6859164. [CrossRef] [PubMed]

17. Rinninella, E.; Cintoni, M.; Raoul, P.; Pozzo, C.; Strippoli, A.; Bria, E.; Tortora, G.; Gasbarrini, A.; Mele, M.C. Effects of nutritional interventions on nutritional status in patients with gastric cancer: A systematic review and meta-analysis of randomized controlled trials. Clin. Nutr. ESPEN 2020, 38, 28-42. [CrossRef]

18. Hamaguchi, Y.; Kaido, T.; Okumura, S.; Kobayashi, A.; Shirai, H.; Yagi, S.; Kamo, N.; Okajima, H.; Uemoto, S. Impact of Skeletal Muscle Mass Index, Intramuscular Adipose Tissue Content, and Visceral to Subcutaneous Adipose Tissue Area Ratio on Early Mortality of Living Donor Liver Transplantation. Transplantation 2017, 101, 565-574. [CrossRef] [PubMed] 
19. Waki, Y.; Irino, T.; Makuuchi, R.; Notsu, A.; Kamiya, S.; Tanizawa, Y.; Bando, E.; Kawamura, T.; Terashima, M. Impact of Preoperative Skeletal Muscle Quality Measurement on Long-Term Survival After Curative Gastrectomy for Locally Advanced Gastric Cancer. World J. Surg. 2019, 43, 3083-3093. [CrossRef]

20. Ebadi, M.; Martin, L.; Ghosh, S.; Field, C.J.; Lehner, R.; Baracos, E.V.; Mazurak, V.C. Subcutaneous adiposity is an independent predictor of mortality in cancer patients. Br. J. Cancer 2017, 117, 148-155. [CrossRef] [PubMed]

21. Lin, J.X.; Lin, J.P.; Xie, J.; Wang, J.; Lu, J.; Chen, Q.; Cao, L.; Lin, M.; Tu, R.; Zheng, C.; et al. Prognostic Value and Association of Sarcopenia and Systemic Inflammation for Patients with Gastric Cancer Following Radical Gastrectomy. Oncologist 2019, 24. [CrossRef] [PubMed]

22. Sugiyama, K.; Narita, Y.; Mitani, S.; Honda, K.; Masuishi, T.; Taniguchi, H.; Kadowaki, S.; Ura, T.; Ando, M.; Tajika, M.; et al. Baseline Sarcopenia and Skeletal Muscle Loss During Chemotherapy Affect Survival Outcomes in Metastatic Gastric Cancer. Anticancer. Res. 2018, 38, 5859-5866. [CrossRef] [PubMed]

23. Rinninella, E.; Cintoni, M.; Raoul, P.; Pozzo, C.; Strippoli, A.; Bria, E.; Tortora, G.; Gasbarrini, A.; Mele, M.C. Muscle mass, assessed at diagnosis by L3-CT scan as a prognostic marker of clinical outcomes in patients with gastric cancer: A systematic review and meta-analysis. Clin. Nutr. 2020, 39, 2045-2054. [CrossRef]

24. Dijksterhuis, W.P.M.; Pruijt, M.J.; Van Der Woude, S.O.; Klaassen, R.; Kurk, S.A.; Van Oijen, M.G.H.; Van Laarhoven, H.W.M. Association between body composition, survival, and toxicity in advanced esophagogastric cancer patients receiving palliative chemotherapy. J. Cachex Sarcopenia Muscle 2019, 10, 199-206. [CrossRef]

25. Choi, Y.; Oh, D.-Y.; Kim, T.-Y.; Lee, K.-H.; Han, S.-W.; Im, S.-A.; Bang, Y.-J. Skeletal Muscle Depletion Predicts the Prognosis of Patients with Advanced Pancreatic Cancer Undergoing Palliative Chemotherapy, Independent of Body Mass Index. PLoS ONE 2015, 10, e0139749. [CrossRef]

26. Blauwhoff-Buskermolen, S.; Versteeg, K.S.; De Van Der Schueren, M.A.E.; Braver, N.R.D.; Berkhof, J.; Langius, J.A.E.; Verheul, H.M.W. Loss of Muscle Mass During Chemotherapy Is Predictive for Poor Survival of Patients with Metastatic Colorectal Cancer. J. Clin. Oncol. 2016, 34, 1339-1344. [CrossRef]

27. Basile, D.; Parnofiello, A.; Vitale, M.G.; Cortiula, F.; Gerratana, L.; Fanotto, V.; Lisanti, C.; Pelizzari, G.; Ongaro, E.; Bartoletti, M.; et al. The IMPACT study: Early loss of skeletal muscle mass in advanced pancreatic cancer patients. J. Cachex. Sarcopenia Muscle 2019, 10, 368-377. [CrossRef] [PubMed]

28. Rier, H.N.; Jager, A.; Sleijfer, S.; van Rosmalen, J.; Kock, M.C.; Levin, M.-D. Low muscle attenuation is a prognostic factor for survival in metastatic breast cancer patients treated with first line palliative chemotherapy. Breast 2017, 31, 9-15. [CrossRef] [PubMed]

29. Antoun, S.; Lanoy, E.; Iacovelli, R.; Albiges-Sauvin, L.; Loriot, Y.; Merad-Taoufik, M.; Fizazi, K.; Di Palma, M.; Baracos, V.E.; Escudier, B. Skeletal muscle density predicts prognosis in patients with metastatic renal cell carcinoma treated with targeted therapies. Cancer 2013, 119, 3377-3384. [CrossRef] [PubMed]

30. Ataseven, B.; Luengo, T.G.; Du Bois, A.; Waltering, K.-U.; Traut, A.; Heitz, F.; Alesina, P.F.; Prader, S.; Meier, B.; Schneider, S.; et al. Skeletal Muscle Attenuation (Sarcopenia) Predicts Reduced Overall Survival in Patients with Advanced Epithelial Ovarian Cancer Undergoing Primary Debulking Surgery. Ann. Surg. Oncol. 2018, 25, 3372-3379. [CrossRef]

31. Van Dijk, D.; Bakens, M.; Coolsen, M.; Rensen, S.; Van Dam, R.; Bours, M.; Weijenberg, M.; De Jong, C.; Damink, S.O. Reduced survival in pancreatic cancer patients with low muscle attenuation index. HPB 2016, 18, e756. [CrossRef]

32. Feliciano, E.M.C.; Kroenke, C.H.; Meyerhardt, J.A.; Prado, C.M.; Bradshaw, P.T.; Kwan, M.L.; Xiao, J.; Alexeeff, S.; Corley, D.; Weltzien, E.; et al. Association of Systemic Inflammation and Sarcopenia with Survival in Nonmetastatic Colorectal Cancer: Results From the C SCANS Study. JAMA Oncol. 2017, 3, e172319. [CrossRef] [PubMed]

33. Kim, E.Y.; Kim, Y.S.; Seo, J.-Y.; Park, I.; Ahn, H.K.; Jeong, Y.M.; Kim, J.H.; Kim, N. The Relationship between Sarcopenia and Systemic Inflammatory Response for Cancer Cachexia in Small Cell Lung Cancer. PLoS ONE 2016, 11, e0161125. [CrossRef] [PubMed]

34. Da Fonseca, G.W.P.; Farkas, J.; Dora, E.; Von Haehling, S.; Lainscak, M. Cancer Cachexia and Related Metabolic Dysfunction. Int. J. Mol. Sci. 2020, 21, 2321. [CrossRef] [PubMed]

35. Caccialanza, R.; Cereda, E.; Caraccia, M.; Klersy, C.; Nardi, M.; Cappello, S.; Borioli, V.; Turri, A.; Imarisio, I.; Lasagna, A.; et al. Early 7-day supplemental parenteral nutrition improves body composition and muscle strength in hypophagic cancer patients at nutritional risk. Clin. Nutr. 2018, 37, S14. [CrossRef]

36. De Waele, E.; Mattens, S.; Honoré, P.M.; Spapen, H.; De Grève, J.; Pen, J.J. Nutrition therapy in cachectic cancer patients. The Tight Caloric Control (TiCaCo) pilot trial. Appetite 2015, 91, 298-301. [CrossRef]

37. Kapoor, N.D.; Twining, P.K.; Groot, O.Q.; Pielkenrood, B.J.; Bongers, M.E.R.; Newman, E.T.; Verlaan, J.J.; Schwab, J.H. Adipose tissue density on CT as a prognostic factor in patients with cancer: A systematic review. Acta Oncol. 2020, 59, 1488-1495. [CrossRef]

38. Okumura, S.; Kaido, T.; Hamaguchi, Y.; Kobayashi, A.; Shirai, H.; Yao, S.; Yagi, S.; Kamo, N.; Hatano, E.; Okajima, H.; et al. Visceral Adiposity and Sarcopenic Visceral Obesity are Associated with Poor Prognosis After Resection of Pancreatic Cancer. Ann. Surg. Oncol. 2017, 24, 3732-3740. [CrossRef]

39. Okumura, S.; Kaido, T.; Hamaguchi, Y.; Kobayashi, A.; Shirai, H.; Fujimoto, Y.; Iida, T.; Yagi, S.; Taura, K.; Hatano, E.; et al. Impact of Skeletal Muscle Mass, Muscle Quality, and Visceral Adiposity on Outcomes Following Resection of Intrahepatic Cholangiocarcinoma. Ann. Surg. Oncol. 2016, 24, 1037-1045. [CrossRef] 
40. Park, H.S.; Kim, H.S.; Beom, S.H.; Rha, S.Y.; Chung, H.C.; Kim, J.H.; Chun, Y.J.; Lee, S.W.; Choe, E.-A.; Heo, S.J.; et al. Marked Loss of Muscle, Visceral Fat, or Subcutaneous Fat After Gastrectomy Predicts Poor Survival in Advanced Gastric Cancer: Single-Center Study from the CLASSIC Trial. Ann. Surg. Oncol. 2018, 25, 3222-3230. [CrossRef]

41. Guiu, B.; Petit, J.M.; Bonnetain, F.; Ladoire, S.; Guiu, S.; Cercueil, J.-P.; Krausé, D.; Hillon, P.; Borg, C.; Chauffert, B.; et al. Visceral fat area is an independent predictive biomarker of outcome after first-line bevacizumab-based treatment in metastatic colorectal cancer. Gut 2009, 59, 341-347. [CrossRef] [PubMed]

42. Brandl, A. Prognostischer Einfluss von Fettgewebsdichte und Muskelmasse beim fortgeschrittenen kolorektalen Karzinom. Prognostic value of adipose tissue and muscle mass in advanced colorectal cancer. Coloproctology 2019, 41, 218-219. [CrossRef] 